Cambodia, ${ }^{3}$ Queen Elizabeth Hospital,

Birmingham, ${ }^{4}$ Gloucestershire Royal Hospital

Learning Objectives: To understand that, in Cambodia, cholesteatoma is often at an advanced stage at presentation. Extrapolation from geographic data suggests that there are barriers to access in remote locations.

Introduction: We have recently instigated the first continual program for tympanomastoid surgery in Cambodia at the Children's Surgical Centre, Phnom Penh. We provide care for adults and children, and cover a population of 15 million individuals, who have previously had no access to otological surgery. We set out to ascertain the severity of disease presenting to us, and proxy measures of access to care, through a retrospective review of patient records.

Methods: We reviewed operative records of all patients undergoing tympanomastoid surgery between February 2014 and March 2016. We recorded the extent of disease, the presence of ossicular erosion, and the location and extent of erosion of the temporal bone. We compared our findings to those reported in the literature. We also used the home address of those presenting to our services to calculate the distance travelled to our centre, and compared this to the population density of each region.

Results: We retrieved records of 74 cases. Erosion of the facial canal, lateral semicircular canal, tegmen, and the ossicles is more extensive and common than reported in previous epidemiological studies. Very extensive disease is also recorded, including post-aural fistulae or abscesses, erosion into the parotid gland, exposure of the sigmoid sinus, and erosion into the internal auditory meatus. Many people had suffered for years before seeking medical care. People living near to our centre were over-represented in our cohort.

Conclusions: Cholesteatoma in Cambodia is at an advanced stage compared to that presenting in the developed world. The epidemiology of those presenting to our centre suggests that awareness of, and access to care is a significant issue for those in remote locations. This is one of the first studies to report on cholesteatoma epidemiology in the developing world, and the findings give impetus to efforts to develop infrastructure to support ear care across the developing world.

doi:10.1017/S0022215116007192

\section{ID: IP223}

Early Detection of Residual

Cholesteatomas by Color Mapped Fusion

Imaging and Removal by Transcanal

Endoscopic Ear Surgery

\section{Presenting Author: Akiko Saitoh}

Akiko Saitoh $^{1}$, Tomoo Watanabe ${ }^{1}$, Tsukasa Ito $^{2}$,

Takatoshi Furukawa ${ }^{2}$, Kazunori Futai ${ }^{3}$, Toshinori Kubota, Masafumi Kanoto ${ }^{5}$, Yuuki Toyoguchi ${ }^{5}$, Takaaki Hosoya ${ }^{5}$, Seiji Kakehata ${ }^{2}$

${ }^{1}$ Department of Otolaryngology, Yamagata

University faculty of Medicine, ${ }^{2}$ Department of
Otolaryngology, Head and Neck Surgery,

Yamagata University Faculty of Medicine,

${ }^{3}$ Yamagata City Hospital Saiseikan, ${ }^{4}$ Yamagata

Prefectural Shinjyo Hospital, ${ }^{5}$ Department of

Diagnostic Radiology, Yamagata University

Faculty of Medicine

Learning Objectives: To demonstrate that CMFI is a reliable diagnostic modality for not only preoperatively identifying cholesteatomas but also postoperatively identifying earlystage residual cholesteatomas.

Introduction: Residual cholesteatomas have been difficult to accurately detect at an early stage during follow-up examinations of patients whom had previously undergone surgery for removal of a primary cholesteatoma. Typically shadows will appear on a CT scan, but cannot be confirmed as a residual cholesteatoma until a second CT scan is taken several months later. This second CT is then compared to the first CT scan to determine whether the shadow has increased in size, thus strongly suggesting the presence of a cholesteatoma. However, color mapped fusion imaging (CMFI) can be used to immediately evaluate such shadows. If a shadow shows up as a red area, the shadow is likely to be a residual cholesteatoma and can be immediately removed. Thus CMFI is useful in the postoperative follow-up evaluations of patients for residual cholesteatomas.

Patients and Methods: Ninety patients who had undergone the removal of a primary acquired middle ear cholesteatoma and were undergoing postoperative follow-up evaluations for residual cholesteatomas at 6-month intervals. Each patient initially underwent a CT scan. If a shadow was found which suggested the presence of a residual cholesteatoma, a CMFI was taken to determine whether the shadow was actually a cholesteatoma. This CMFI is created by combining a 1-mm thin slice non-EPI DWI with MR cisternography (MRC).

Results: Shadows were found on the initial CT scan in 68/90 patients. The presence of a residual cholesteatoma was strongly suggested in 5/68 patients based on the CMFI. These 5 patients all underwent surgery for cholesteatoma removal. The CMFI evaluations for these patients were compared to the intraoperative findings. All 5 patients were found to have a residual cholesteatoma in the same anatomical location as indicated by the CMFI and these cholesteatomas were all successfully removed.

Conclusion: CMFI is a reliable diagnostic modality for postoperatively identifying early-stage residual cholesteatomas.

doi:10.1017/S0022215116007209

\section{ID: IP224}

Endoscopic Ear Surgery for the Removal of Residual and Recurrent Cholesteatomas

\section{Presenting Author: Tomoo Watanabe}

Tomoo Watanabe ${ }^{1}$, Tsukasa Ito ${ }^{1}$, Takatoshi Furukawa ${ }^{1}$, Kazunori Futai $^{2}$, Toshinori Kubota ${ }^{3}$, Seiji Kakehata ${ }^{1}$ 